Revue d'histoire de l'Amérique française

REVUE D.HISTOIRE DE L'AMÉRIQUE FRANÇAISE

\title{
Séjour de Champlain à Brouage en 1630
}

\section{Delafosse}

Volume 9, numéro 4, mars 1956

URI : https://id.erudit.org/iderudit/301793ar

DOI : https://doi.org/10.7202/301793ar

Aller au sommaire du numéro

Éditeur(s)

Institut d'histoire de l'Amérique française

ISSN

0035-2357 (imprimé)

1492-1383 (numérique)

Découvrir la revue

Citer ce document

Delafosse, M. (1956). Séjour de Champlain à Brouage en 1630. Revue d'histoire de l'Amérique française, 9(4), 571-578. https://doi.org/10.7202/301793ar d'utilisation que vous pouvez consulter en ligne.

https://apropos.erudit.org/fr/usagers/politique-dutilisation/ 


\section{DOCUMENTS INÉDITS}

\section{SEJOUR DE CHAMPLAIN A BROUAGE EN 1630}

La liste complète des actes notariés concernant Champlain, qui à ma connaissance n'a jamais été publiée, serait assez courte. Composée surtout d'actes parisiens ou rouennais tels ceux que nous venons de présenter à l'exposition France et Canada, elle n'aurait compris jusqu'à maintenant aucun document de Brouage. Les deux textes publiés ici combleront cette lacune et ajouteront un détail à la vie de Champlain. Je les ai trouvés récemment dans les minutes du notaire brouageais Gouin restées jusqu'alors inexplorées; ils n'apportent sans doute aucune révélation sensationnelle, mais rien de ce qui touche au fondateur de Québec n'étant indifférent, ils me paraissent mériter la publication.

Ce sont deux ventes faites par Champlain le 27 septembre 1630 de deux maisons qu'il possédait encore à Brouage, l'une assez grande cédée pour 1150 livres tournois à la fabrique de l'église Notre-Dame, l'autre plus petite vendue 700 Livres à un marchand. Les ventes n'indiquent malheureusement pas l'origine de la propriété. Elles sont chargées de la rente ordinaire, 50 sous par «place», due au roi comme seigneur de Brouage ou plutôt de la chatellenie voisine d'Hiers (Les mots « rente noble » ne doivent naturellement pas faire illusion). Leur emplacement est précisé par le nom des rues mais je ne suis pas actuellement en état de le situer dans le Brouage d'aujourd'hui, si tentant soit-il de pouvoir ajouter une «maison de Champlain » à la prétendue «maison de Marie Mancini ».

Ces documents nous montrent donc que Champlain est venu à Brouage lors de son dernier séjour en France et qu'il y était encore propriétaire. Ce n'est pas sans intérêt; en effet, même les historiens de Brouage naturellement enclins à renforcer les liens entre Champlain et Brouage croyaient qu'il n'y était pas revenu et qu'il n'y avait pas gardé de biens; c'était par exemple 
l'opinion du Docteur Vincent auteur d'une bonne histoire de Brouage trop peu connue. On a même tendance aujourd'hui à mettre en question le qualificatif «de Brouage» que Champlain pourtant, s'est lui-même donné, et certes il faut reconnaître qu'il subsiste bien des obscurités. Ainsi le nom de Champlain ne me paraît pas se retrouver comme nom de famille saintongeais et il ne serait pas impossible que son père soit venu d'ailleurs puisque Brouage fut formé d'éléments très divers. Quoi qu'il en soit voici deux actes qui nous présentent Champlain propriétaire à Brouage, au milieu des habitants de la petite ville qui achètent une de ses maisons pour y loger leur curé. Brouage est à cette date un centre encore prospère, animé par la construction des belles fortifications et par le commerce du sel. Les minutes Gouin apportent justement à ce sujet des renseignements nouveaux qui complètent notre connaissance de Brouage en ce début de siècle.

La liquidation de ses immeubles fut-elle le seul motif de la venue de Champlain qui se procure ainsi 1850 livres, somme non négligeable en 1630 ? Remarquons d'abord que Charles Leber sieur du Carlo, à qui suivant l'acte de 1625 Champlain portait «bonne et vraie amour naturelle » était mort: Deux actes du notaire Gouin, l'un du 23 mars 1629 où étant commissaire de l'artillerie, il afferme son droit de visite des navires à Brouage, l'autre du 26 Novembre 1629 citant le curateur «des enfants mineurs de feu Charles Leber sieur du Carlo » permettent de situer sa mort entre ces deux dates. Constatons d'autre part que le port de Brouage avait encore un reste d'activité. Toujours devant Gouin est passé le 20 janvier 1629 le contrat d'affrètement du «Louis », 50 tonneaux du Chateau d'Oleron, maître Vincent Bonnenfant d'Olonne, que Jean Tuffet, un des Directeurs de la Compagnie de la Nouvelle France envoyait «au grand fleuve Saint Laurent jusqu'au lieu de Québec ». Le rapport de mer devant l'Amirauté de La Rochelle (B. $5654 \mathrm{n}^{\circ}$ 70) nous apprend d'ailleurs que le Louis n'alla pas jusqu'à Québec; retenu en rade de la Rochelle par Rasilly qui se préparait lui aussi à partir pour le Canada avec plusieurs vaisseaux, il n'atteignit le Canada qu'après la chute de Québec et revint avec un navire de 
Bordeaux. L'année même où Champlain était à Brouage le 19 novembre 1630 , le commis de Jean Tuffet donnait, par acte de Gouin, un acompte de 400 livres tournois à Jean Guillard maître du navire le Petit Saint Jean de Bordeaux « à présent de retour du voyage de la Nouvelle France étant au Havre de Brouage ». A cette date Champlain me paraît avoir quitté Brouage car il ne signe pas, le 4 novembre, au contrat de mariage de l'architecte François Chauvin où figurent les notables de la ville. Il n'est pourtant pas téméraire de supposer que ce navire revenant de Canada peut aussi avoir attiré Champlain dans ce port qui est encore à cette date un centre de relations avec la Nouvelle France.

Où alla-t-il ensuite ? J'ai vainement cherché trace de son passage à la Rochelle dans la même année, bien qu'un acte du notaire Cousseau nous y montre le 18 mars Louis Gaigneur (membre de cette famille de marchands qui commerça si activement par la suite avec Québec), faisant charger une barque de biscuits, farines et raisins pour la Compagnie de la NouvelleFrance suivant les ordres de Tuffet. Retrouvera-t-on d'autres actes notariés sur Champlain ? Les richesses des minutiers parisiens déposés aux Archives Nationales sont telles qu'on peut toujours espérer y faire des découvertes; il reste aussi des minutes de notaires de Brouage actuellement conservées à Marennes qui devront être explorées, sans grande chance de succès cependant.

M. Delafosse, Archiviste en chef de la Charente-Maritime, France.

N.B. - Avec cette livraison de la Revue, votre abonnement prend fin pour l'année 1955-1956. Vous vous ferez, sans doute, un devoir de le renouveler le plus tôt possible. Le prix du réabonnement est de $\$ 5.00$. 


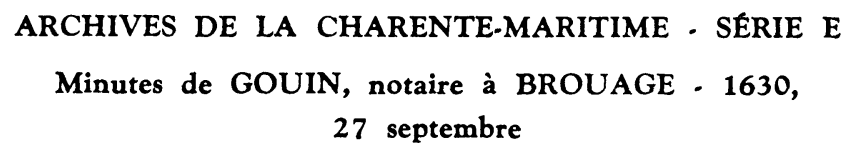

Par devant le nottaire tabellion roial gardenottes heredictere en Xainctonge soubs signé et en presance des tesmoings bas nommés et escripts ont este presant et personnellements estabis en droict Samuel de Champelain cappictaine pour le Roy en la marine commendant pour Monseigneur le Cardinal en la Nouvelle France dicte Canada d'une part et Gregoire Le Camus et Antoine Naudin fabriqueurs de l'esglize Notre Dame de Brouaige et marchants demeurants audit Brouaige d'autre part et noble homme Louis Bonnin Sieur de la Guilloterye Recepveur pour le Roy de ladite ville de Brouaige et isle de Xaintonge demeurant audit Brouaige encorre d'autre part et Jean de Loubert escuyer sieur de Marchis lieutenant d'une compagnie en la garnisson dudit Brouaige et Maistre François Juillard lieutenant en ladjudicature roial dudit lieu fermier de la fabrique de ladite ville encore d'autre part, entre lesquelles partyes en presence et de l'advis et consantement de sieures Pierre Gombaud Pierre Aubouin Anthoine Fremureau Eslie Lepveraud Jean Gille sieur des Houmeaux Jean Grain Jean Castel Jean Boubet Pol Mousnier Me Jean Papin Jean Rocquet David Grain Pierre Girardeau faisant la plus grande majeure part de ladite ville de Brouaige et chastellenie d'Hieres, ont esté faicts et accordées ces présentes par lesquelles ledit sieur de Champelain a vendu ceddé quitté remis dellaissé allienné et transporté vand cedde quicte remect dellaisse allienne et transporte par ces presantes ausdicts Naudin et Cammus audit nom de fabricqueurs de ladite eglise Notre Dame de Brouaige à ce presents et stippullants c'est assavoir une maisson basse couverte de tuille scittuee en ceste ville de Brouaige contenant deux places avec toutes et chascunes ses appartenances et deppandances de fonds solle entree issue basse court jardin agoust desgoust vues servitudes et autre sans rien en resserver ni retenir scittuee et confronté d'ung bout et par devant à la ruhe sableuze d'autre bout et par le derrière à la ruhe heureuse d'ung costé à la ruhe Saint Jean et d'autre costé aux maissons de [blanc] Lafontaine maître pattissier et la vefve Pier [?] Guilfelt, ladicte maisson tenneue à rente du Roy à causse de sa chastelanie d'Hieres a raisson de cents sols tournois de rente noble directe et fontierre paiable par chascun 
an à chascun jour et feste de Saint Jean Baptiste laquelle maison et appartenance susdite ledit sieur de Champellain s'en est entierremant desmis desvestu et dessaisy du tout en tout en a vestu et saisy lesdits Naudin et Cammus ausdits noms et au proffict de ladicte esglize Notre Dame de Brouaige pour par iceux en jouir uzer faire et disposser à leur plaissir et vollonté sans contredict leur en donnant et laissant toutte bonne et libre pocession et saizine par ces presantes consantant qu'ils la prenne reelle actuelle et corporelle sur les lieux comme ils advizeront soict en sa presance ou abesance, faicte ladicte vente cession et transeport pour et moyennant le prix et somme de unze cents cinquente livres tournois laquelle somme a esté baillée et paiée presantement et contant audit sieur de Champelain sçavoir la somme de sept cents cinquente livres par ledit sieur Bonnin comme heritier et donnatere de deffunt noble homme Samuel Bonnin son frere vivent sieur de la Guilloterie et recepveur pour le Roy des presentes ylles en conséquence du testament dudit feu Bonnin receu Guillet notaire roial en datte du dixiesme janvier mil six cens six et la somme de quatre cents livres tournois par lesdits sieurs de Marchis et Juilhard sur les deniers restans du prix de la ferme des marois de la fabrique dudit Brouaige et Hieres que lesdits sieurs de Marchis et Juilhard tienne en vertu du contrat d'icelle receu de mesme notaire que ces presentes en dabte du vingt quatriesme de janvier dernier, lesdits paiements faicts en bonne especes de pistolles escus d'or quars d'escus et autre bonne monnoie parfaissant ladite somme de unze cents cinquente livres tournois que ledit sieur de Champelain a veue contee prinze receue embourcé emportee et s'en contante renonçant a toute erreur de compte et exeption de pecune en tient quicte lesdits acquereurs et tous autres, en faveur duquel present marché ledit sieur de Champelain demeure quicte des reparations faictes en ladite maison comme parreillement lesdits acquereurs des fermes et locations de tout le temps passé et attant ledit sieur de Champelain a promis toute bonne et loiale garrentie de ladite maisson et appartenance en cas d'esviction en payent dessormais et a l'advenir la rente seigneurrialle annuelle et perpetuelle qui est la somme de cent solz a la recepte de la presente ville à chaque jour et feste de Saint Jean Baptiste lesquels fabriqueurs et habittans de ladite ville de Brouaige et chastellanie d'Hieres ont déclaré qu'ils onct faict ladite acquissition pour et au proffict de la fabrique dudit Brouaige et Hieres et pour emploier une partie de ladite ville de Brouaige et Chastellanie d'Hieres et une autre partie en bastiments propres pour en tirer locations et faire fonds de recette pour ladite 
fabrique laquelle maisson et appartenance et recette qui en proviendera demeure speciallement obligee aux prieres services et charges portées et contenues par le testament dudict deffunct maître Samuel Bonnin cy dessus rapporté et generallement tous et chascuns les biens de ladite fabrique presents et advenir et ledit sieur Louis Bonnin quitté et deschargé de ladicte somme principalle de sept cents cinquante livres portée par ledit testament ensemble des arreraiges de rentes ou interest d'icelle de tout le temps passé pour lesquels arreraiges ou interest il a paié contant es mains desdits fabriqueurs la somme de soixante dix huit livres deux sols six deniers tournois pour une année et huict mois restant deubs, comme aussy lesdicts sieures de Marchis et Juillard demeurent quictes de ladite somme de quatre cents livres qui leur sera desduicte sur ce qu'ilz peuvent debvoir de ladite ferme de maroix. Tout ce que dessus a esté ainsy voullu consenty agréé acordé stippullé et accepté par les parties de part et d'autre lesquels à l'entretien à peine de tous despands domaiges et interests ont obligé et hipottecqué sçavoir ledit sieur de Champellain pour ladicte garrentye tous et chascunes ses biens meubles et immeubles presants et advenir et lesdicts fabriqueurs et habitans pour la descharge desdicts sieurs Bonnin du Marchis et Juillard et pour l'observation des charges prieres et service portée par le testament dudit feu sieur Bonnin tous et chascuns les biens de ladicte fabricque et speciallement la susdicte maisson et appartenance sans que l'obligation sepecialle desroge a la generalle ny au contraire renonçant a tout cas contraire, promettant et jurant leur foix de jamais n'y contrevenir donct vollontairement ils ont été juges et condempnés par le nottaire roial soubssigné o dhue submissions. Faict et passé à Brouaige en la maisson de Monsieur de La Rocheallard en presance de Maître Jean Jolly et Vincent Beluchau dict la Vallée praticiens et Ollivier Paineau soldact de la garnizon demeurans audit Brouaige le vingt septiesme jour de septembre mil six cens trente.

$$
\text { (Signé): } \quad \text { CHAMPLAIN. }
$$

DE LOUBERT. BONNYN. GOMBAULD. JULHARD. Anthoine NAUDIN. J. GILLES. AUBOUYN. LE CAMUS. David GRAIN. LEVRAUD. PAPIN. FREMUREAU. J. GRAIN. BOUBET. Pol MOUNIR. ROQUET. GIRARDEAU. JOLLY. CASTET. BESSONNEAU. BELLUCHEAU. 
Par devant le nottaire tabellion roial Gardenot heredictaire en Xaintonge soubs signé et en presance des tesmoingts bas nommés et escrits ont estés presents et personnellements establis en droict Samuel de Champellain cappictaine pour le Roy en la marine comandant pour Monseigneur le Cardinal en la Nouvelle France dict Canada d'une part et Jean de Loubert escuier sieur du Marchis et lieutenant d'une compaignie en la garnison de Brouaige faissant pour et au nom de sieur Jean Grain marchant d'autre part, lequel sieur de Champelain de sa bonne vollonté a vandu cedé quicté transporté vand cede quicte transporte par ces presentes audit sieur du Marchis audit nom stippullant et accepant c'est assavoir une maisson faicte à faix et à plancher avecq toutes et chascunes ses appartenances et depandance quelconques de fons solle entree yssues bassecourt jardin veue et servitude scittuee et assise en ceste ville de Brouaige tenue à rente du Roy à causse de sa chatalanie d'Hieres au debvoir de cinquente sols tournois par chascun an au jour et feste de Saint Jean Baptiste et ce confronte d'ung bout a la ruhe Teigneusse d'autre bouct à la ruhe Boivin d'ung costé à la maisson de la veuve Bouchaud la meuraille commune entre deux et d'autre costé à la maisson du Sauvaige aussy la meurraille commune entre deulx de laquelle dite maisson et appartenance susdite ledit sieur de Champellain s'est entieremant desmis desvestu et dessaizy du touct en tout et en a vestu et saizy ledit sieur du Marchis audit nom pour par luy en jouir uzer faire et dispozer à son plaissir et vollonté sans nul contredict luy en donnant et laissant la pocession libre et vacquente par ces presentes et consantant la reelle, faicte ladicte vente et cession et transeport pour et moyenant le prix et somme de sept cents livres tournois laquelle ledit sieur du Marchis a baillee et payée presentement et contant audit sieur de Champelain en bonne espesses de pistolles escus d'or et autre bonne monnoie parfaissant ladite somme de sept cents livres tournois que ledit sieur de Champlain a eue contee receue embource emportée et s'en contante renonçant a toute erreur de compte et eseption de pecune en tient quicte ledit sieur du Marchis lequel paiement ledit sieur du Marchis avoit fait pour ledit sieur Grain et des deniers d'icelluy Grain sans que ledit sieur du Marchis en aict debource aucune chosse des siens et attant ledit sieur de Champelain a promis garentir et indempnizer et deffendre ladite maisson et appartenance audit sieur du Marchis et es siens desormais et pour l'advenir de tous troubles debtes ypottecques arreraiges de rente et empeschemants quelconques en payant 
desormais et pour l'advenir lesdicts cinquente soubs de rente noble a la recepte dudit Brouaige sans rien plus touct ce que dessus a este ainsy voullu consanty agrée accordé stippullé et accepté par lesdites parties de part et d'autre lesquels à l'entretien à peine de tous despands dommaiges et interrests onct obligé tous leurs biens meubles et immeubles presans et advenir quelconques, Renonçant etc ... Promettans etc ... Jugés et condemnés etc... Soubmis etc... Faict et passé audit Brouaige en la maisson de Monsieur de la Rocheallard avent midy en presance de Maître Jean Papin notaire roial et sieur Antoine Naudin marchent et Vincent Belluchau dict la Vallée demeurant ledit Papin à Hières et lesdits Naudin et Belluchau audit Brouaige le vingt septiesme septembre mil six cens trente (Signé) CHAMPELAIN DE LOUBERT PAPIN Anthoine NAUDIN BELLUCHAU -

Le même jour procès-verbal de prise de possession de la maison par le sieur du Marchis en présence du locataire Etienne Olivier et le 14 février 1635 approbation de l'achat par David Grain fils. 